http://jmscr.igmpublication.org/home/

ISSN (e)-2347-176x ISSN (p) 2455-0450

crossref DOI: https://dx.doi.org/10.18535/jmscr/v7i7.97

Journal Of Medical Science And Clinical Research

IGM Publication

An Official Publication of IGM Publication

\title{
A descriptive study to assess the self reported level of Cultural Competence to care for a Transcultural population among Nurses in a tertiary care hospital
}

Authors

\author{
Radhika Das, Punita A Sharma
}

\begin{abstract}
Background: The world today is becoming smaller and is no longer separated by differences in food, language or religion. Recent advances in work, technology and medicine has made people frequently travel round the globe. A nurse today has to be efficient in care of not only one kind of patients, but those from every corner of the world with different food habits, religion or ways of living. When a nurse provides care that includes knowledge, attitudes, and skills that support caring for people across different languages and cultures she is called Culturally Competent. Nurses serving in the Armed forces serve across the terrains of the country and even abroad. Giving culturally relevant care is an essential part of their functions, as they commonly care for patients who are of different cultural background than theirs. Transcultural nursing though being a distinct nursing speciality has today become the need of every nurse which focuses on global cultures and comparative cultural caring, health, and nursing phenomena.

Objective of the study was to assess the self reported level of cultural competence to care for a transcultural population among nurses in a tertiary care hospital.

Methods/Design: Quantitative non experimental study design was followed. A descriptive cross sectional study was conducted among selected nurses of a tertiary care hospital. 100 nurses were selected following convenient sampling. Tool had two sections with questions of sociodemographical variables and Nurse Cultural competence Scale (NCCS) regarding cultural competence among the nurses in the tertiary care hospital.

Results: Cultural Knowledge among the nurses was 336, Cultural Awareness was 243, Cultural Sensitivity was 258, and Cultural Skill among the selected nurses was 325 out of a subtotal of 500. The results reveal that the selected nurses had adequate knowledge on Culture and skills required for effective care, but needed to improve on Cultural awareness and Sensitivity.

Keywords: Transcultural Nursing, Cultural Competence, Nurses.
\end{abstract}

What is already known about the topic?

1. Patients demand care as per their religious and cultural beliefs

2. Nurses are required to provide culturally competent and adaptive care

3. Nurses skilled in transcultural nursing produced better output in terms of patient and hospital outcomes
What this paper adds?

1. This study measures the self reported level of different components of transcultural nursing among the selected nurses.

2. This study throws light on the aspects to be improved upon among the nurses to improve transcultural nursing. 


\section{Introduction}

The world today is a small place as people are no longer tied down by threads of religion, food or family ties. Distances across nations are covered in a jiffy and it has become natural for people to often travel or settle in places other than their native country. Health requirements, vacation, boredom, lack of job opportunities, physical and environmental hazards are few of the reason that humans shift and settle in greener pastures. Populations who have shifted often imbibe the ways of living of the host country, but try and retain their native food habits and religious beliefs to a large extent. The health needs of such a population also reflect their inherent traditions and values. It is therefore imperative that the health system of a country be inclusive of the needs, cultural practises and requirements of its foreign population.

Culture influences not only health practices but also how the healthcare provider and the patient perceive illness. Becoming aware of how culture influences individual behaviour and thinking allows you to plan the best care for your patients. ${ }^{1}$ A nurse who is not culturally competent or has the wrong attitude to non natives may find it difficult to provide efficient nursing care and this may result in dissatisfaction in self and in the patients. A nurse is said to be culturally competent when she is able to provide care that includes knowledge, attitudes, and skills that support caring for people across different languages and cultures. ${ }^{2}$ It is important in today's scenario that nurses are trained to evolve into a culturally competent care giver.

While serving in the Armed Forces, Nurses form a part of the health care team that works across a variety of terrains. Nurses are posted across the length and breadth of the country and at times even abroad on foreign missions. They provide care to the combatants and their families at the place of their posting away from their native places. Providing timely and efficient care requires the Nurses to be tolerant and aware of various cultural practises and beliefs, otherwise they might be considered insensitive and indifferent, possibly even incompetent. She has to be careful to avoid stereotyping patients and assuming that all patients have similar needs and beliefs. $^{3}$

\section{Background}

Cultural competence is the ability of healthcare providers and organizations to meet the cultural, social, and linguistic needs of their patients. Patients need to feel that healthcare providers respect their beliefs, customs, values, language, and traditions and are more likely to communicate freely and honestly, which can, in turn, reduce disparities in healthcare and improve patient outcomes. ${ }^{4}$ In the military setup, when the soldiers or their families are away from their near and dear ones, it is highly comforting for the sick ones to know that the nursing care provided is inclusive of their beliefs and practises. Small measures like change in menu planning, providing space for praying or allowing of carrying out of harmless religious practises in the hospitals can have long lasting health benefits for our military population.

\section{Materials and methods}

Quantitative non experimental study design was followed. A descriptive cross sectional study was conducted among selected nurses working in a tertiary care hospital. Accessible population was 350 nurses out of which 100 were selected following convenient sampling. Following principles of research ethics and obtaining consent of the participating nurses, a self administered tool, was administered by the researcher at the area of work of the nurses. The nurses took an average of 10 minutes to complete the questionnaire under the supervision of the researcher and doubts if any were cleared by the researcher.

Tool - had two sections. Section I - included Socio demographic variables of the Nurses and Section II was the structured standardised questionnaire. The face and content validity of the tool was done by 07experts from the field of 
Psychiatry and Nursing Education, and changes were made in the tool as per the suggestions.

A pilot study conducted on 15 nurses to check the feasibility of the study, proved it to be feasible to conduct in the selected population. The socio demographic variables included were -

- Age of the Nurses

- Years of services completed

- Professional qualification/ specialization

- Have you cared for patients of cultural background different from yours?

- Religion

- If ever posted/worked in own native place

- Have you heard of the term Transcultural Nursing.

- If yes - Have you any attended any class /symposium/ workshop on Transcultural Nursing?.

Nurse Cultural competent Scale (NCCS) measured the level of cultural competence among nurses. It includes four domains: cultural awareness referring to the nurses' consciousness of the similarities and differences between individual and others' cultural context, and recognition of one's own prejudice with ten items measured in this domain. Cultural knowledge refers to the nurses' knowledge of obtaining information about diverse groups and their culture, such as health beliefs, cultural values; nine items measured domain. Cultural sensitivity refers to the nurses' appreciation of the client's beliefs, valuing their culture and respecting its influence on client's behaviours with eight items and Cultural skills refer to the nurses' ability to carry out the cultural assessment for client, communicate with client by using resources and provide appropriate care without individual prejudice with 14 items. ${ }^{5}$

\section{Results}

100 samples participated in the study. 31 were in the age group of $22-30 \mathrm{yrs}(31.0 \%), 38$ (38.0 $\%$ ) in the age group of $31-40$ yrs and 23 (23.0 $\%$ ) were in the age group of $41-50 \mathrm{yrs}$, and 08 $(8.0 \%)$ were in the age group of 51 years and above. The total number of samples upto $5 \mathrm{yrs}$ of service were 37 (37.0\%), with 6- 10 yrs of service were $20(20.0 \%)$ and 11- 15 yrs of service were $22(22.0 \%)$ and with 16- 20 yrs of service were 12 $(12 \%)$ and those with more than 20 yrs of service were $08(08 \%)$.

As per educational qualification 68(68\%) were undergraduate nurses, 20 (20\%) were graduate nurses, and 12(12\%) were post graduate nurses and specialised in a particular field of nursing. 93(93\%) of nurses had cared for patients from cultural background different than self, and only $23(23 \%)$ of nurses had ever been posted to their native place. Out of the total $87.5(87.5 \%)$ have heard of the term Transcultural nursing and $12(12 \%)$ have attended a class/conference on the same. The distribution of the scores on Cultural Competence Scale of the Nurses is as follows -

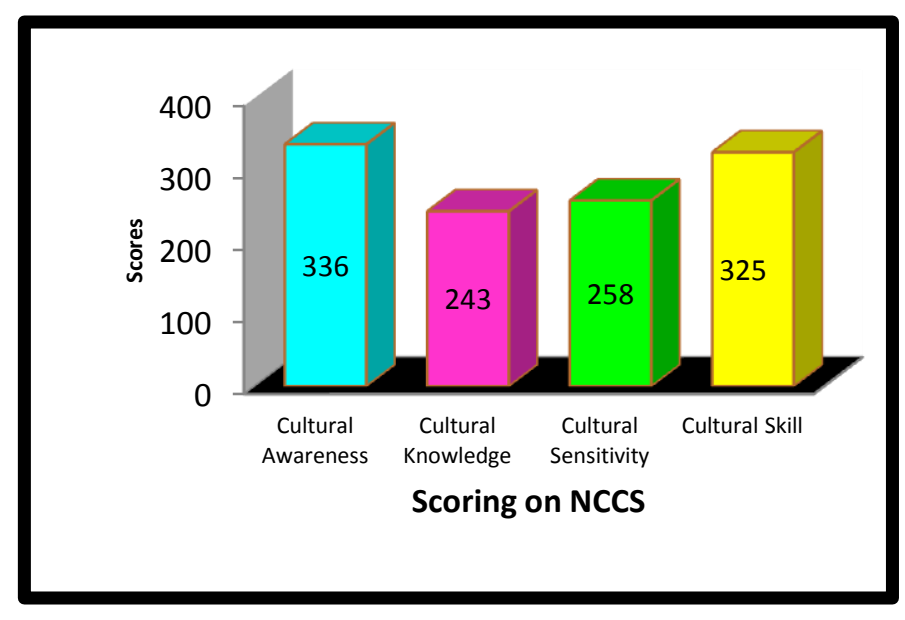

\section{Discussion}

Cultural competence provides many benefits for healthcare professionals and healthcare organizations. Social benefits include increased mutual respect between healthcare workers and patients, increased trust, and promotion of patient responsibility and empowerment in their own healthcare.

In the present study it is seen that on comparison of various aspect of cultural competence, Cultural awareness (336) and skill (325) are adequate among the nurses whereas Cultural Knowledge (243) and sensitivity (258) needs to be improved. If nurses are more sensitive to the needs of the patient and develop more interest in gaining 
knowledge of cultures of patients under her care, it improves the patient health and satisfaction outcomes.

Relationship with Socio demographic variableThe total scores of cultural competency were higher in the nurses of age 22-30 yrs with component of Cultural Knowledge prevailing high. Component of Cultural Skill was higher in age group of $41-50$ yrs. Nurses with work experience of $16 \mathrm{yrs}$ and above scored higher in the Cultural knowledge component.

A study done in 2016 among Nursing faculty revealed that the nurses were moderately culture competent and that there was a need to train the nurses in cultural competency. ${ }^{6}$ When patients feel heard and understood by their healthcare providers, they are more likely to participate in preventive health care and less likely to miss health appointments. This can reduce medical errors and related legal costs for healthcare facilities, and it can improve health outcomes for patients.

\section{Rationale}

The aim of this project is to identify the self reported level of cultural competence among nurses with an aim to increase awareness among administrators and educators and to include education on transcultural nursing as an integral part of nursing curriculum.

\section{Conclusion}

With increase in diverse patient population, the demand for improved cultural competency among the nurses also increases. Cultural competence provides many benefits for healthcare professionals and organizations. Social benefits include increased mutual respect between healthcare workers and patients, increased trust, and promotion of patient responsibility and empowerment in their own healthcare.

The goals of transcultural nursing is to give culturally congruent nursing care, and to provide culture specific and universal nursing care practices for the health and well-being of people or to aid them in facing adverse human conditions, illness or death in culturally meaningful ways Ultimately, a culturally competent organization can help reduce health disparities among patient populations-still a common problem today.

To improve cultural competence and self-efficacy while caring for patients of different cultures, programs should be developed or curricula should be amended to incorporate diversity care techniques, rather than only providing cultural awareness.

\section{Conflict of Interest - Nil}

\section{Reference}

1. file:///C:/Users/HP/Downloads/The_import ance_of_cultural_competence.1.pdf

2. Seeleman C, Suurmond J, Stronks K. Cultural competence: a conceptual framework for teaching and learning. Med Educ. 2009;43(3):229-237..

3. Understanding Transcultural Nursing. Nursing 2005: January 2005 - Volume 35 Issue - P 14,16,18,21,23

4. https://www.medcomrn.com/index.php/arti cles/healthcare-cultural-competence

5. Self-Eff. icacy and Cultural Competency Assessment

of the Associate Degree Nursing Student. Deborah Smith Hartman. Walden University. 2017

6. Assessment of cultural competence in Texas nursing faculty. Marzilli C . Nurse Education Today 45 (2016) 225-229. http://dx.doi.org/10.1016/j.nedt.2016.08.02 1. 